\title{
Editors prefer articles with good originality reports and visuals
}

\author{
Hakan Demirci \\ Department of Family Medicine, University of Health Sciences, Bursa Yuksek Ihtisas Training and Research \\ Hospital, Bursa, Turkey
}

Nowadays, whoever I speak to conveys similar wishes. They say, "I hope the European Journal of Human Health will be included in the SCI index soon". Of course, it is our greatest wish to be included in important academic indexes as soon as possible, but we are aware that much effort is needed on this path. Achieving quality research articles is our first goal. So, what determines quality? That's where writers and editors cross paths. In fact, if there is an honest and high-quality research article, publishing this article is the most basic task of an editor.

In the literature, there are educational articles that guide academic authors, such as 'reasons for rejecting an article', allowing researchers to test themselves (1-3). Some wellknown journals publish this kind of information even in the instructions for authors' section (4). Undoubtedly, an original hypothesis is at the heart of scientific research. Re-testing a subject that has been done thousands of times will obviously lower your paper's chances. More than this, if your sample size is low, it will be difficult for your article to stand out from the hundreds of research articles that reach the editor. It is certainly in your favor to do a power analysis while you are still planning your research. An adequate number of figures and graphs will definitely bring you one step further towards a possible acceptance.

The researcher does not have to confirm the $\mathrm{H}_{1}$ hypothesis, but he has to pass on his findings to the reader in all their transparency. Using misleading or fictitious information will eventually give you away. This means that you will be looking for another job in the continuation of your life. Being reliable is a very important virtue in the scientific arena. So how will the editor trust researchers? This is a big mystery. Perhaps the author's previous work on the subject can convince the editor. Low similarity in your article is another factor that strengthens trust. If the similarity reports are sine qua non for editors, they should also be for researchers. The upper limits of acceptability of similarity are controversial (5\% or 20\%), but it should be remembered that the lower the better.

Use of fluent language is also a topic that reviewers and editors are very focused on. Language editing is now a sector in its own right. The level of a researcher's language skills may not be known, or what suits their budget, but a bad and boring narrative can exclude even research with very high-quality data. Using simple language and avoiding unnecessary word decorations are essential to a scientific article. Statistical analysis and the adequacy and intelligibility of your tables are also vital for the reader and therefore for the editor. You can be an exceptional observer, you can be a very successful clinician, but not knowing enough statistics should not be an obstacle for you. For this reason, a successful language editor and a reliable statistician are the most valuable partners for both authors and editors.

In conclusion, the European Journal of Human Health is looking for powerful articles written in an original, fluent language, with a low level of similarity and findings supported by visuals. Our editorial team considers it a duty to publish articles that meet these criteria. 


\section{References}

1. Thrower, P. Eight reasons I rejected your article. A journal editor reveals the top reasons so many manuscripts don't make it to the peer review process [blog post], 2012.

2. Khadilkar, S. S. (2018). Rejection blues: why do research papers get rejected?. The Journal of Obstetrics and Gynecology of India. 2018;68(4):239-241.

3. Ehara, S., \& Takahashi, K. Reasons for rejection of manuscripts submitted to AJR by international authors. American journal of Roentgenology. 2007;188(2):113-116.

4. Common reasons for rejection Available at: https://www.springernature.com/gp/authors/campaigns/how-to-submit-a-journalarticle-manuscript/common-rejection-reasons Access Date: 28.12.2021. 